\title{
On Topology Preservation for Hexagonal Parallel Thinning Algorithms
}

\author{
Péter Kardos and Kálmán Palágyi \\ Department of Image Processing and Computer Graphics, \\ University of Szeged, Hungary \\ \{pkardos,palagyi\}@inf.u-szeged.hu
}

\begin{abstract}
Topology preservation is the key concept in parallel thinning algorithms on any sampling schemes. This paper establishes some sufficient conditions for parallel thinning algorithms working on hexagonal grids (or triangular lattices) to preserve topology. By these results, various thinning (and shrinking to a residue) algorithms can be verified. To illustrate the usefulness of our sufficient conditions, we propose a new parallel thinning algorithm and prove its topological correctness.
\end{abstract}

Keywords: hexagonal grids, parallel reduction operators, topology preservation, thinning.

\section{Introduction}

Thinning is an iterative layer-by-layer erosion until skeleton-like shape features of binary objects are left 2511]. A thinning algorithm should preserve topology, that is, the produced output pictures should be topologically equivalent to the input ones for all possible digital binary pictures 4. Parallel thinning algorithms are composed of parallel reduction operators (i.e., some object points having value of " 1 " in a binary picture that satisfy certain topological and geometric constrains are changed to "0" ones simultaneously) [2].

Sufficient conditions for topology preserving parallel reduction operators working on orthogonal grids have been given in 2D [2,3|8] and 3D [36]. These results provide methods of verifying that a parallel thinning (and shrinking to a residue) algorithm preserves topology.

Digital pictures on non-orthogonal grids have been studied by a number of authors 477. A hexagonal grid is formed by a tessellation of regular hexagons. By duality, it corresponds to the triangular lattice, where the points are the centers of that hexagons, see Fig. 1. Hexagonal grids have a major advantage over the orthogonal ones. In 2D orthogonal/rectangular grids, the 8-adjacency relation is frequently used [4], where the length of diagonal moves is $\sqrt{2} \cdot a$ if the length of the horizontal and vertical moves is $a$. In hexagonal sampling scheme, each pixel is surrounded by six equidistant nearest neighbors. This results in a less ambiguous connectivity structure and in a better angular resolution compared to the rectangular case. 
The majority of existing thinning algorithms work on orthogonal grids 2[11. However, some parallel thinning methods were also proposed for hexagonal grids 19191012.

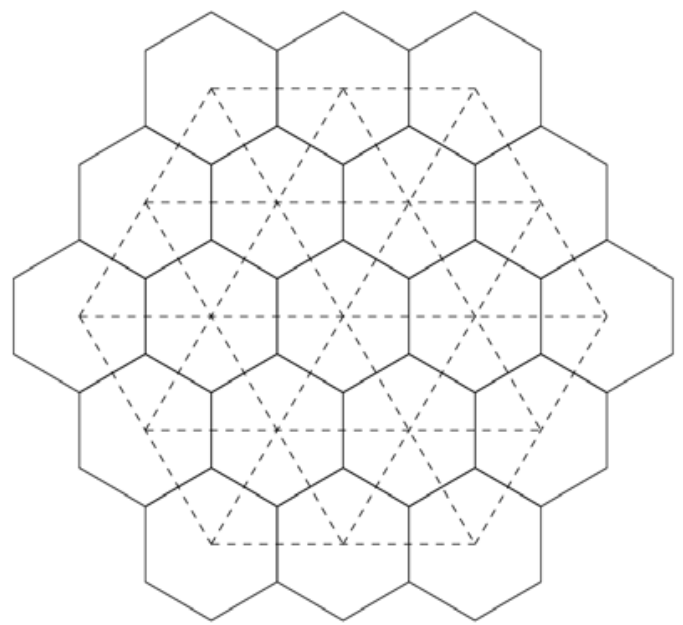

Fig. 1. A hexagonal grid and the corresponding triangular lattice

In this work we establish sufficient conditions for topology preserving parallel reduction operators in binary digital images sampled on hexagonal grids. By our results, various thinning (and shrinking to a residue) algorithms can be proved to be topology preserving.

The rest of this paper is organized as follows. Section 2 summarizes the basic notions of 2D digital topology. In Section 3, we discuss the mentioned sufficient conditions. To illustrate the usefulness of these conditions, we propose a new parallel subiteration-based thinning algorithm in Section 4 and prove that it is topology preserving for $(6,6)$ pictures.

\section{Basic Notions}

Let us consider a hexagonal grid denoted by $H$, and let $p$ be a pixel in $H$. Let us denote $N_{6}(p)$ the set of pixels being 6-adjacent to pixel $p$ and let $N_{6}^{*}(p)=$ $N_{6}(p) \backslash\{p\}$. Figure 2 shows the 6 -neighbors of a point $p$ denoted by $N_{6}(p)$. The pixel denoted by $p_{i}$ is called as the $i$-th neighbor of the central pixel $p$.

The sequence $S$ of distinct pixels $\left\langle x_{0}, x_{1}, \ldots, x_{n}\right\rangle$ is called a 6 -path of length $n$ from pixel $x_{0}$ to pixel $x_{n}$ in a non-empty set of pixels $X$ if each pixel of the sequence is in $X$ and $x_{i}$ is 6 -adjacent to $x_{i-1}$ for each $1 \leq i \leq n$. Note that a single pixel is a 6 -path of length 0 . In the special case when $x_{0}=x_{n}$ in $S$, we talk about a 6 -cycle, and the sequence $\left\langle x_{i}, x_{i+1}, \ldots, x_{j}\right\rangle(0 \leq i \leq j \leq n)$ is called a subpath of $S$. Two pixels are said to be 6 -connected in set $X$ if there is a 6 -path in $X$ between them. 

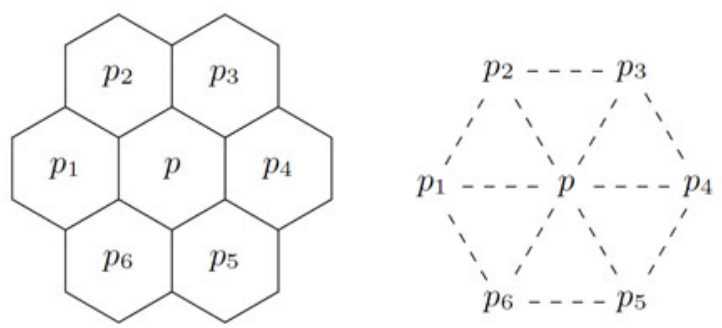

Fig. 2. Indexing scheme for the elements of $N_{6}(p)$ on hexagonal grid (left) and triangular lattice (right)

Based on the concept of digital pictures as reviewed in 4 , we define the $2 D$ binary $(6,6)$ digital picture as a quadruple $\mathcal{P}=(H, 6,6, B)$. The elements of $H$ are called the pixels of $\mathcal{P}$. Each pixel in $B \subseteq H$ is called a black pixel and has a value of 1 . Each pixel in $H \backslash B$ is called a white pixel and the value of 0 is assigned to it. 6-adjacency is associated with both black and white pixels. A black component or an object is a maximal 6 -connected set of pixels in $B$, while a white component or a cavity is a maximal 6-connected set of pixels in $H \backslash B$. An object composed of three mutually 6-adjacent black pixels is a unit triangle.

Let us denote $C_{6}(p)$ the number of black components in picture $(H, 6,6, B \cap$ $\left.N_{6}^{*}(p)\right)$. A black pixel is called a border pixel in a $(6,6)$ picture if it is 6 -adjacent to at least one white pixel. A black pixel $p$ is called an $i$-border pixel in a $(6,6)$ picture if its $i$-th neighbor (denoted by $p_{i}$ in Fig. 2) is a white pixel $(1 \leq i \leq 6)$. A black pixel $p$ is called an end pixel in a $(6,6)$ picture if it is 6 -adjacent to exactly one black pixel.

A reduction operator transforms a binary picture only by changing some black pixels to white ones (which is referred to as the deletion of 1's). A parallel reduction operator deletes all pixels satisfying its condition simultaneously. A $2 \mathrm{D}$ reduction operator does not preserve topology [3] if any black component is split or is completely deleted, any white component is merged with another white component, or a new white component is created.

A simple pixel is a black pixel whose deletion is a topology preserving reduction [4. Let $\mathcal{P}$ be a $(6,6)$ picture. The set of black pixels $D=\left\{d_{1}, \ldots, d_{k}\right\}$ is called a simple set of $\mathcal{P}$ if $D$ can be arranged in a sequence $\left\langle d_{i_{1}}, \ldots, d_{i_{k}}\right\rangle$ in which $d_{i_{1}}$ is simple and each $d_{i_{j}}$ is simple after $\left\{d_{i_{1}}, \ldots, d_{i_{j-1}}\right\}$ is deleted from $\mathcal{P}$, for $j=2, \ldots, k$. (By definition, let the empty set be simple.)

\section{Sufficient Conditions for Topology Preserving Parallel Reductions}

In this section we discuss two important relationships for topology preservation in $(6,6)$ pictures. We will prove in Theorem 1 that the simplicity of a pixel in a $(6,6)$ picture is a local property, and based on this rule we will give sufficient conditions for a parallel reduction operator to preserve topology in Theorem 2 , 
Theorem 1. Black pixel $p$ in picture $(H, 6,6, B)$ is simple if and only if both of the following conditions are satisfied:

1. $p$ is a border pixel.

2. $C_{6}(p)=1$.

Proof. First we show indirectly that if Conditions 1 and 2 are satisfied, then $p$ is simple. Let us suppose that the above conditions hold for $p$, and if we delete $p$, an object will be split into more components. Then there must be two pixels $q, r \in B$ such that all 6-paths in $B$ between $q$ and $r$ contain $p$. This implies that all 6-paths in $B$ between $q$ and $r$ contain a subpath $\left\langle p_{i}, p, p_{j}\right\rangle(i, j \in\{1,2, \ldots, 6\}, i \neq j)$, as well (see Fig. 3 a). However, this contradicts Condition 2 , by which $p_{i}$ and $p_{j}$ are also 6 -connected in picture $(H, 6,6, B \backslash\{p\})$. Hence, no object is split by the removal of $p$.

Now let us suppose that if we delete $p$ then a white component is merged with another white component (or with the background). Then, there exist two white pixels $q, r \in H \backslash B$ such that all 6-paths in $(H \backslash B) \cup\{p\}$ between $q$ and $r$ contain $p$. Thus, all 6-paths in $H \backslash B$ from $q$ to $r$ contain a subpath $\left\langle p_{i}, p, p_{j}\right\rangle$ $(i, j \in\{1,2, \ldots, 6\}, i \neq j)$, as well. Let us consider the case when $i=1 . j \neq 2$, or else the path $\left\langle p_{i}, p_{j}\right\rangle$ would be also a subpath of a 6 -path from $q$ to $r$, but this subpath does not contain $p$. If $j=3$ (see Fig. 3b), then, according to our assumption, both of the 6-paths $\left\langle p_{1}, p_{2}, p_{3}\right\rangle$ and $\left\langle p_{3}, p_{4}, p_{5}, p_{6}, p_{1}\right\rangle$ must contain a black pixel. Similarly, if $j=4$ (see Fig. [3r), then the 6-paths $\left\langle p_{1}, p_{2}, p_{3}, p_{4}\right\rangle$ and $\left\langle p_{4}, p_{5}, p_{6}, p_{1}\right\rangle$ must contain a black pixel. But for both of the possible cases we come into a contradiction with Condition 2. Because of the symmetry of the neighborhood of $p$ composed by the elements of $N_{6}(p)$ (see Fig. 2), we can derive similar results if we change the values of the indexes $i, j$. Hence, no white component is merged with another white component (or with the background) by the removal of $p$.

If a new white component would be arisen when deleting $p$, then $p$ could not be a border pixel in picture $(H, 6,6, B)$, but this means a contradiction with Condition 1.

If an object would be deleted by the removal of $p$, then this would mean that $p$ is an isolated object pixel, which implies $C_{6}(p)=0$. However, this is a contradiction with Condition 2 .

For all possible cases we came into a contradiction with Condition 1 or 2, therefore, $p$ is a simple pixel. Now we will also indirectly show that if $p$ is simple, then Conditions 1 and 2 hold.

Let us suppose that $p$ is a simple pixel but at least one from the above mentioned conditions fails to hold. If $p$ would not be a border pixel, then a new white component would be arisen by the removal of $p$, which can not happen because of the simplicity of $p$, therefore, $C_{6}(p) \neq 1$ must be satisfied.

As $p$ is a simple pixel, the deletion of $p$ does not lead to splitting an object into more components. This can only happen if between any two pixels $p_{i}, p_{j}$ $(i, j \in\{1,2, \ldots, 6\}, i \neq j)$ there exists a 6 -path in $B$ not containing $p$. If we add $p$ to this path, then we obviously get a 6 -cycle in $B$. Let us denote this 6 -cycle 
$P_{1}$ (see the continuous line in Fig. 3 $\mathrm{d}$ ). From the property $C_{6}(p) \neq 1$ follows that such a 6-cycle does not only contain pixels from $N_{6}^{*}(p)$, hence there exist such $q, r \in(H \backslash B) \cap N_{6}^{*}(p)$ for which the 6-path from $p_{i}$ to $p_{j}$ in set $N_{6}^{*}(p) \backslash\{r\}$ contains $q$ and the 6 -path from $p_{i}$ to $p_{j}$ in set $N_{6}^{*}(p) \backslash\{q\}$ contains $r$. There must be a 6-path between $q$ and $r$ in the set $H \backslash B$, or else a white component would be merged with another white component (or with the background) by the removal of $p$, which is not possible. If we add $p$ to this path, we get a 6-cycle in $H \backslash B \cup\{p\}$ that we denote by $P_{2}$ (see the dotted line in Fig. $3 \mathrm{~d}$ ).

Let $S_{P k}=\left\{x \mid x\right.$ is contained in $\left.P_{k}\right\}(k \in\{1,2\})$. It is easy to see that both of the pictures $\left(H, 6,6, H \backslash S_{P_{1}}\right)$ and $\left(H, 6,6, H \backslash S_{P_{2}}\right)$ contain exactly two objects. One of the objects in picture $\left(H, 6,6, H \backslash S_{P_{1}}\right)$ necessarily contains all elements of set $S_{P_{2}} \backslash\{p\}$, or else $q$ and $r$ would not be 6 -connected in picture $(H, 6,6, H \backslash B)$, hence the removal of $p$ would reduce the number of white components. It is obvious that this object in $\left(H, 6,6, H \backslash S_{P_{1}}\right)$ fully contains one of the objects in $\left(H, 6,6, H \backslash S_{P_{2}}\right)$, which we will denote by $O . p_{i}$ or $p_{j}$ must be a member of $O$, or else $q$ and $r$ would be also 6 -connected in picture $\left(H, 6,6,(H \backslash B) \cap N_{6}^{*}(p)\right)$, hence one of the 6 -paths from $p_{i}$ to $p_{j}$ in $N_{6}^{*}(p)$ should contain both $q$ and $r$, which contradicts to our assumptions on $q, r$. However, $p_{i}$ and $p_{j}$ are contained in cycle $P_{1}$, which means, $p_{i}, p_{j} \in S_{P_{1}}$, therefore, none of these pixels may be a member of $O$. According to the derived contradiction, Conditions 1 and 2 hold for simple pixel $p$.

Lemma 1. Let $p$ and $q$ two 6 -adjacent simple pixels in picture $\mathcal{P}=(H, 6,6, B)$. The following statements are equivalent:

1. $p$ is simple in picture $(H, 6,6, B \backslash\{q\})$, or $q$ is simple in picture $(H, 6,6, B \backslash\{p\})$.

2. $N_{6}^{*}(p) \cap N_{6}^{*}(q) \cap(H \backslash B)$ contains exactly one element.

Proof. The first part of the proof will be carried out indirectly. Let us suppose that Statement 1 is true but the set $S=N_{6}^{*}(p) \cap N_{6}^{*}(q) \cap(H \backslash B)$ contains two elements or $S=\emptyset$. First, let us examine the case when $S$ contains two elements, i.e., both common 6-neighbors of $p$ and $q$ are white in picture $\mathcal{P}$. As both $p$ and $q$ are simple in this picture, $C_{6}(p)=C_{6}(q)=1$ holds by Theorem 1 which is possible only if $N_{6}^{*}(p) \cap B=\{q\}$ and $N_{6}^{*}(q) \cap B=\{p\}$. But in this case, $p$ is an isolated object pixel in picture $(H, 6,6, B \backslash\{q\})$, thus the removal of $p$ would also result in the removal of an object. Hence, $p$ is not a simple pixel in this case, which contradicts the condition on $p$. As a conclusion, $S=\emptyset$, i.e., both pixels in $N_{6}^{*}(p) \cap N_{6}^{*}(q)$ must be black. Let us denote these pixels by $r_{1}$ and $r_{2}$. By Statement 1 and Theorem 1. $C_{6}(p)=1$ in picture $(H, 6,6, B \backslash\{q\})$ or $C_{6}(q)=1$ in picture $(H, 6,6, B \backslash\{p\})$. Because of the symmetry of the image part covered by the pixels of $N_{6}^{*}(p) \cap N_{6}^{*}(q)$, it is sufficient to only examine the first of these possible situations. In this case, there must be a 6-path from $r_{1}$ and $r_{2}$ in $N_{6}^{*}(p) \cap(B \backslash\{q\})$. This path necessarily contains all elements of $N_{6}^{*}(p) \backslash\{q\}$, from which follows that all 6 -neighbors of $p$ is black in picture $\mathcal{P}$. But in this case, $p$ can not be a border pixel, which is a contradiction with our initial assumption. Hence, if Statement 1 is satisfied, then Statement 2 must be also fulfilled. 
Now let us suppose that Statement 2 is true. We show that $p$ is simple in picture $(H, 6,6, B \backslash\{q\})$. As one of the 6-neighbors of $q$ contained in $N_{6}^{*}(p)$ is white in picture $\mathcal{P}$, the removal of $q$ from $\mathcal{P}$ does not result in splitting of any component in picture $\left(H, 6,6, B \cap N_{6}^{*}(p)\right)$. Furthermore, as one of the 6 -neighbors of $q$ contained in $N_{6}^{*}(p)$ is black, the removal of $q$ from $\mathcal{P}$ does not lead to the removal of any object from the latter picture. Hence, $C_{6}(p)=1$ still holds, which means, Statement 1 is also satisfied by Theorem 1 .

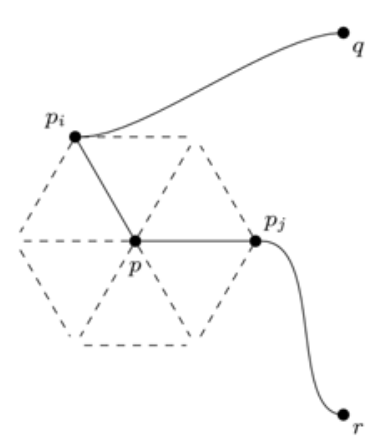

a

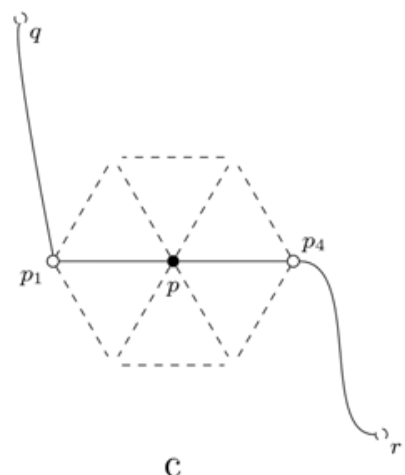

C

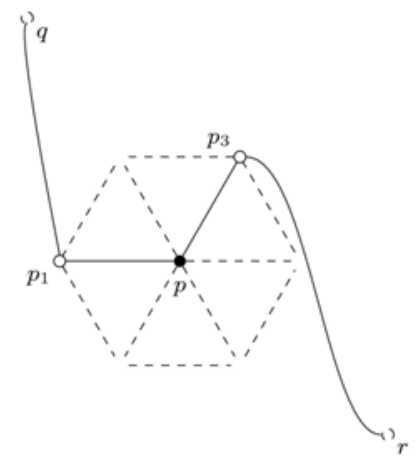

$\mathrm{b}$

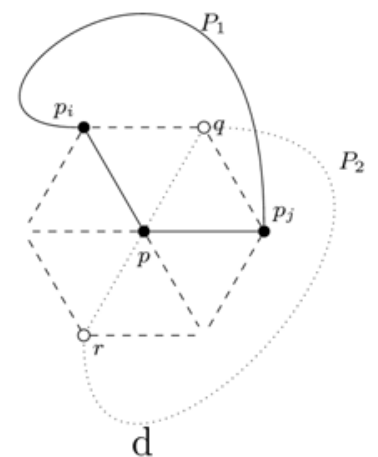

Fig. 3. The examined cases in the proof of Theorem 1

Lemma 2. Let $\mathcal{O}$ be a parallel reduction operator, and let $S$ be the set of black pixels removed by $\mathcal{O}$ from an arbitrary picture $\mathcal{P}=(H, 6,6, B) . \mathcal{O}$ is topologypreserving, if for any pixel $p \in S$ and for any set $Q \subseteq S \cap N_{6}^{*}(p), p$ is simple in picture $(H, 6,6, B \backslash Q)$.

Proof. Let us suppose that the condition in the lemma is fulfilled on $\mathcal{O}$. As $\emptyset \subseteq S \cap N_{6}^{*}(p)$, any pixel $p \in S$ is simple in $\mathcal{P}$. Let $S=S_{n}=\left\{s_{1}, s_{2}, \ldots, s_{n}\right\}$ $\left(n \in \mathbb{N}^{+}\right)$, furthermore, let $S_{i}=\left\{s_{1}, s_{2}, \cdots, s_{i}\right\}(1 \leq i \leq n)$. It is sufficient to see that $S_{n}$ is a simple set in $\mathcal{P}$. 
The proof will be done by induction on $i . s_{1}$ is simple in $\mathcal{P}$, hence set $S_{1}=\left\{s_{1}\right\}$ is also simple in $\mathcal{P}$. Let us suppose that set $S_{k}$ is simple in $\mathcal{P}(1 \leq k<n)$. $S_{k+1}=$ $S_{k} \cup\left\{s_{k+1}\right\}$, thus we have to prove that $s_{k+1}$ is simple in picture $\left(H, 6,6, B \backslash S_{k}\right)$.

It is useful to make a distinction between the pixels in $S_{k}$ being and not-being 6-neighbors of $s_{k+1}$, as by Theorem 1. only the deletion of pixels in $N_{6}^{*}\left(s_{k+1}\right)$ may influence the simplicity of $s_{k+1}$. Therefore, $s_{k+1}$ remains simple in picture $\mathcal{P}_{k}=\left(H, 6,6, B \backslash\left(S_{k} \backslash N_{6}^{*}\left(s_{k+1}\right)\right)\right)$. We only have to examine what happens if we remove some black 6 -neighbors of $s_{k+1}$ in $\mathcal{P}_{k}$. Let $Q_{k}=S_{k} \cap N_{6}^{*}\left(s_{k+1}\right)$ (i.e., $Q_{k}$ contains exactly that pixels of $S_{k}$ which are 6-neighbors of $s_{k+1}$ ). As $S_{k} \subseteq S$, $Q_{k}=S_{k} \cap N_{6}^{*}\left(s_{k+1}\right) \subseteq S \cap N_{6}^{*}\left(s_{k+1}\right)$ holds, too. It is easy to see that if we remove $Q_{k}$ from the set of black pixels in $\mathcal{P}_{k}$, then we obtain the reduced set $B \backslash S_{k}$. Thus, if we apply the condition on $\mathcal{O}$ for picture $\mathcal{P}_{k}$, we get that $s_{k+1}$ is simple in picture $\left(H, 6,6, B \backslash S_{k}\right)$.

Theorem 2. A parallel reduction operator $\mathcal{O}$ is topology-preserving in picture $\mathcal{P}=(H, 6,6, B)$, if all of the following conditions hold:

1. Only simple pixels are deleted by $\mathcal{O}$.

2. If $\mathcal{O}$ removes two 6 -adjacent pixels $p, q$, then $p$ is simple in picture $(H, 6,6$, $B \backslash\{q\})$, or $q$ is simple in picture $(H, 6,6, B \backslash\{p\})$ (i.e., $\{p, q\}$ is a simple set).

3. $\mathcal{O}$ does not delete completely any black component contained in a unit triangle.

Proof. Let us suppose that $\mathcal{O}$ satisfies Conditions 1-3, and let us denote $S$ the set of black pixels deleted by $\mathcal{O}$. By Lemma 2 it is sufficient to show that, for any $p \in S$ and for any $Q \subseteq S \cap N_{6}^{*}(p), p$ is simple in picture $(H, 6,6, B \backslash Q)$. This is obviously satisfied for $Q=\emptyset$ by Condition 1 , thus we have only to examine the case when $Q \neq \emptyset$.

If $N_{6}^{*}(p) \cap S=\{q\}$, i.e., $\mathcal{O}$ deletes exactly 1 black pixel from the 6 -neighbors of $p$, then $Q=\{q\}$ must hold, and according to Conditions 1 and $2, p$ is simple in picture $(H, 6,6, B \backslash Q)$.

Now let us assume that $N_{6}^{*}(p) \cap S=\{q, r\}$, i.e., $\mathcal{O}$ deletes exactly 2 black pixels from the 6 -neighbors of $p$. If $Q$ contains only one element, then we can show similarly to the previous case that $p$ is simple in picture $(H, 6,6, B \backslash Q)$. Let us suppose that $Q=\{q, r\}$. The following two cases can be distinguished:

I. $q$ and $r$ are 6-adjacent.

II. $q$ and $r$ are not 6 -adjacent.

First, let us examine Case I. By Conditions 1-2 and by Lemma 1, set $N_{6}^{*}(p) \cap$ $N_{6}^{*}(r) \cap(H \backslash B)$ contains exactly one element, hence the 6-neighbor of $r$ not coinciding with $q$ is white in picture $\left(H, 6,6, N_{6}^{*}(p) \cap B\right)$. It can be similarly derived that the 6-neighbor of $q$ not coinciding with $r$ is white in picture $\left(H, 6,6, N_{6}^{*}(p) \cap\right.$ $B)$. Thus, set $\{q, r\}$ is a black component in picture $\left(H, 6,6, N_{6}^{*}(p) \cap B\right)$. Because of the symmetrical arrangement of $p, q, r$, we can similarly prove that set $\{p, q\}$ is a black component in picture $\left(H, 6,6, N_{6}^{*}(r) \cap B\right)$, and the set 
$\{p, r\}$ is a black component in picture $\left(H, 6,6, N_{6}^{*}(q) \cap B\right)$. As by Theorem 1 , $C_{6}(p)=C_{6}(q)=C_{6}(r)=1$ holds for $\mathcal{P}$, it also follows from the above conclusions that set $\{p, q, r\}$ is not 6-connected with any pixel $s \in B \backslash\left(N_{6}(p) \cup N_{6}(q) \cup N_{6}(r)\right)$ in $B$. Therefore, set $\{p, q, r\}$ is surrounded only by white pixels in $\mathcal{P}$, which means that $\{p, q, r\}$ is a black component in $\mathcal{P}$. However, this contradicts to Condition 3 , hence Case I can not come into question.

Now, let us discuss Case 2. According to Conditions 1-2 and Lemma 1, both of the sets $N_{6}^{*}(p) \cap N_{6}^{*}(q) \cap(H \backslash B)$ and $N_{6}^{*}(p) \cap N_{6}^{*}(r) \cap(H \backslash B)$ contain exactly one element. Obviously, $N_{6}^{*}(p) \cap N_{6}^{*}(r)$ contains two elements. From these also follows that set $N_{6}^{*}(p) \cap N_{6}^{*}(r) \cap B$ contains exactly one element. Let $N_{6}^{*}(p) \cap N_{6}^{*}(r) \cap$ $B=\{s\}$. Based on Conditions 1 and 2, $p$ is simple in picture $(H, 6,6, B \backslash\{q\})$. Furthermore, by Condition $1, r$ is simple in $\mathcal{P}$, thus $C_{6}(r)=1$ holds by Theorem 11 and as $q \notin N_{6}(r), C_{6}(r)=1$ still holds after the removal of $q$, which means that $r$ is simple in picture $(H, 6,6, B \backslash\{q\})$. By examining the possible arrangements of $q$ and $r$, we can conclude that $q \neq s$, therefore the set $N_{6}^{*}(p) \cap N_{6}^{*}(r) \cap$ $(H \backslash(B \backslash\{q\}))$ also contains exactly one element. Hence, by Lemma 1 $p$ is simple in picture $(H, 6,6, B \backslash Q)$.

Finally, we indirectly prove that $\mathcal{O}$ may not delete more than 2 black 6 neighbors of $p$. Let $q_{1}, q_{2}, q_{3} \in N_{6}^{*}(p)$ and let us suppose that $\mathcal{O}$ deletes all the pixels of set $S=\left\{p, q_{1}, q_{2}, q_{3}\right\}$. If a pixel $q_{i}(i \in\{1,2,3\})$ would be 6 -adjacent to every element of $S \backslash\left\{q_{i}\right\}$, then $N_{6}(p) \cap N_{6}\left(q_{i}\right) \cap(H \backslash B)=\emptyset$ would hold. However, this would lead to a contradiction with Lemma 1, hence this case is not possible. In every other cases, it is sure that some pixels $q_{i}$ and $q_{j}(i, j \in\{1,2,3\}, i \neq j)$ are not 6 -connected in picture $\left(H, 6,6, B \cap N_{6}^{*}(p)\right)$, which implies that $C_{6}(p)>1$. But this is not possible as $p$ is a simple pixel in $\mathcal{P}$, which means by Theorem 1 that $C_{6}(p)=1$ holds, thus we came into a contradiction again.

\section{A New Topology Preserving Thinning Algorithm}

In this section we introduce a new parallel thinning algorithm working on hexagonal arrays. The strategy which is used is called subiteration-based [2]: each iteration step is composed of six parallel reduction operators according to the six directions assigned to the six neighbors of a pixel in a hexagonal array. Deletable pixels assigned to the $i$-th subiterations are given by matching template $T_{i}$, see Fig. 4 ( $1 \leq i \leq 6)$.

Algorithm 1 outlines the proposed 6-subiteration parallel thinning algorithm.

It is easy to see that Algorithm 1 cannot delete any end pixel since each template $T_{i}(1 \leq i \leq 6)$ matches black pixels that are 6 -adjacent to more than one black pixels (see Fig. 4). Hence Algorithm 1 can produce medial curves.

In experiments Algorithm 1 was tested on objects of different shapes. Figure 5 presents three illustrative examples.

Now we show that the proposed algorithm is topology preserving. At first, some important properties of pixels that are matched by template $T_{1}$ are stated.

Proposition 1. All pixels deleted by the parallel reduction operator given by template $T_{1}$ are 1-border pixels. 


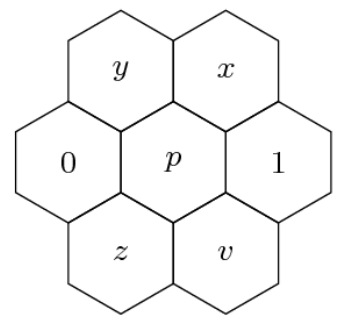

$T_{1}$

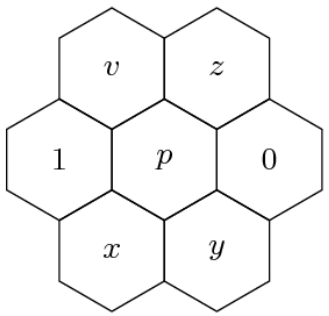

$T_{4}$

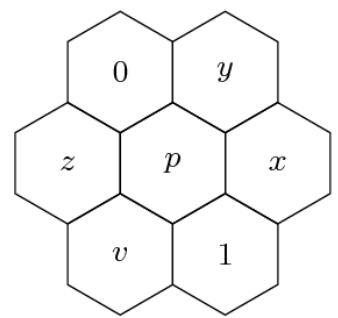

$T_{2}$

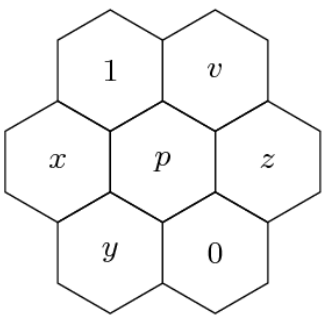

$T_{5}$

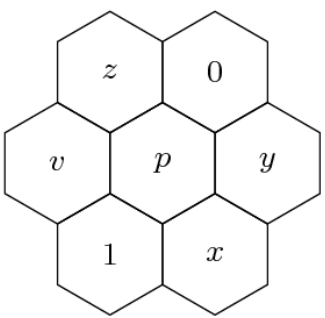

$T_{3}$

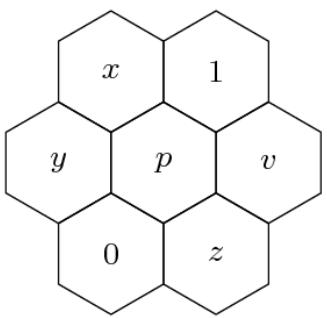

$T_{6}$

Fig. 4. Matching templates of the proposed subiteration-based algorithm. Template $T_{i}$ is assigned to the $i$-th subiteration $(i=1,2, \ldots, 6)$. Notations (for each template): positions marked " $p$ " and " 1 " match two black pixels; position marked " 0 " matches a white pixel; at least one positions marked " $x$ " and " $v$ " matches a black pixel; if position " $y$ " matches a black pixel, then position " $x$ " matches a black pixel, too; if position " $z$ " matches a black pixel, then position " $v$ " matches a black pixel, as well.

This holds since the 1st neighbor of a black pixel matched by $T_{1}$ is a white pixel.

Proposition 2. If a black pixel can be deleted by template $T_{1}$, then its 4 th neighbor cannot be deleted by $T_{1}$.

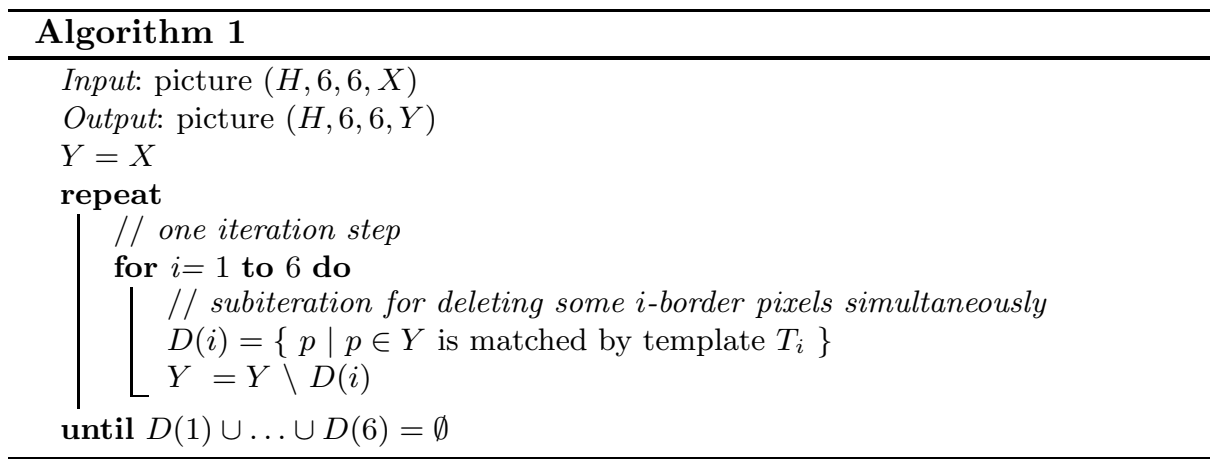



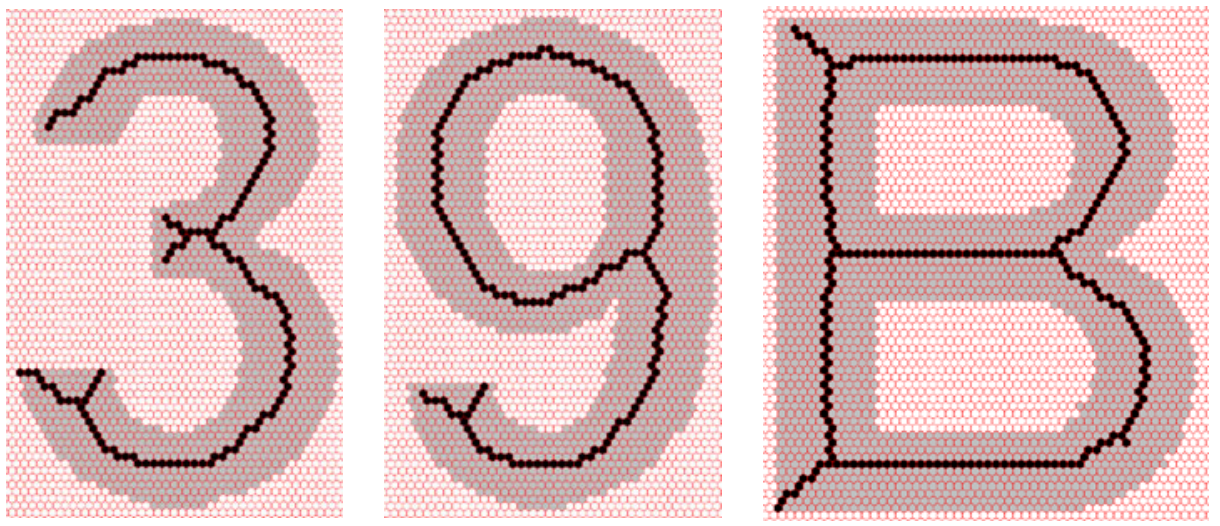

Fig. 5. Thinning of three objects sampled on hexagonal grids. Medial curves produced by Algorithm 1 are superimposed on the original objects.

This holds by Proposition 1 , since if an object pixel matches template $T_{1}$, then the 4th neighbor of that pixel can not be a 1-border pixel, hence that neighbor does not match $T_{1}$.

Theorem 3. Algorithm 1 is topology preserving for $(6,6)$ pictures.

Proof. To prove it, we show that the parallel reduction operator given by template $T_{1}$ satisfies all conditions of Theorem 2

1. Let us examine the simplicity of a pixel $p$ that is matched by template $T_{1}$. The first thing we need to verify that $p$ is a border pixel. This holds by Proposition 1, hence Condition 1 of Theorem 1 is satisfied. To prove that Condition 2 of Theorem 1 holds, we show that $C_{6}(p)=1$ for any $p$ deleted by $T_{1}$. This is fulfilled since if position " $y$ " matches a black pixel, then position " $x$ " matches a black pixel, and if position " $z$ " matches a black pixel, then position " $v$ " matches a black pixel (see Fig. (4). Hence Condition 1 of Theorem 2 is satisfied.

2. It was proved that only simple pixels are deleted by $T_{1}$. To prove that Condition 2 of Theorem 2 holds, we show that for each pixel $p$ deleted by $T_{1}$, $C(p)$ remains 1 after a neighbor of $p$ is deleted by $T_{1}$.

- The 1st neighbor of $p$ is a white pixel.

- The 2nd neighbor of $p$ coincides with the template position " $y$ ". If it can be deleted by $T_{1}$, then the template position " $x$ " matches a black pixel. Hence $C(p)=1$ after the deletion of its 2nd neighbor.

- The 3rd neighbor of $p$ coincides with the template position " $x$ ". If it can be deleted by $T_{1}$, then template position " $y$ " coincides with a white pixel. Hence $C(p)=1$ after the deletion of its 3rd neighbor.

- The 4th neighbor of $p$ is a black pixel. Since $p$ is its 1st neighbor, it cannot deleted by $T_{1}$. 
- The 5th neighbor of $p$ coincides with the template position " $v$ ". If it can be deleted by $T_{1}$, then template position " $z$ " coincides with a white pixel. Hence $C(p)=1$ after the deletion of its 5 th neighbor.

- The 6th neighbor of $p$ coincides with the template position " $z$ ". If it can be deleted by $T_{1}$, then the template position " $v$ " matches a black pixel. Hence $C(p)=1$ after the deletion of its 6 th neighbor.

If pixel $p$ is deleted by $T_{1}$, then $p$ is a 1-border pixel by Proposition 1 . It is obvious that pixel $p$ remains a 1-border pixel after $q \in N_{6}^{*}(p)$ is deleted by $T_{1}$. Since $p$ is a 1-border pixel and $C(p)=1$ after the deletion of $q, p$ remains a simple pixel after $q$ is deleted by $T_{1}$. Hence Condition 2 of Theorem 2 is satisfied.

3. Suppose that pixel $p$ is an element of an arbitrary black component. If $p$ is deleted by $T_{1}$, then its 4 th neighbor $q$ is a black and it cannot be deleted by Proposition 2. Since both pixels $p$ and $q$ are in the same black component, no black component can be deleted completely by $T_{1}$. Hence Condition 3 of Theorem 2 is satisfied.

We proved that the parallel reduction operator given by template $T_{1}$ is topology preserving. The remaining five subiterations of Algorithm 1 given by templates $T_{2}, T_{3}, T_{4}, T_{5}$, and $T_{6}$ are topology preserving operators since these templates can be obtained by rotations of template $T_{1}$. Algorithm 1 is topology preserving since it is composed of topology preserving operators.

\section{Conclusions}

This paper presents a characterization of simple point in $(6,6)$ digital pictures sampled on hexagonal grids (or triangular lattices) and establishes sufficient conditions for topology preserving parallel reduction operators working on $(6,6)$ pictures. By our results, various thinning (and shrinking to a residue) algorithms can be proved to be topology preserving. To illustrate the usefulness of our sufficient conditions, we have proposed a new parallel subiteration-based thinning algorithm and we have proved its topological correctness.

\section{Acknowledgements}

This research was supported by the TÁMOP-4.2.2/08/1/2008-0008 program of the Hungarian National Development Agency, the European Union and the European Regional Development Fund under the grant agreement TÁMOP4.2.1/B-09/1/KONV-2010-0005, and the grant CNK80370 of the National Office for Research and Technology (NKTH) \& the Hungarian Scientific Research Fund (OTKA). 


\section{References}

1. Deutsch, E.S.: Thinning algorithms on rectangular, hexagonal, and triangular arrays. Communications of the ACM 15, 827-837 (1972)

2. Hall, R.W.: Parallel connectivity-preserving thinning algorithms. In: Kong, T.Y., Rosenfeld, A. (eds.) Topological algorithms for digital image processing, pp. 145179. Elsevier, Amsterdam (1996)

3. Kong, T.Y.: On topology preservation in 2-d and 3-d thinning. Int. Journal of Pattern Recognition and Artificial Intelligence 9, 813-844 (1995)

4. Kong, T.Y., Rosenfeld, A.: Digital topology: Introduction and survey. Computer Vision, Graphics, and Image Processing 48, 357-393 (1989)

5. Lam, L., Lee, S.-W., Suen, C.Y.: Thinning methodologies - A comprehensive survey. IEEE Trans. Pattern Analysis and Machine Intelligence 14, 869-885 (1992)

6. Ma, C.M.: On topology preservation in 3D thinning. CVGIP: Image Understanding 59, 328-339 (1994)

7. Marchand-Maillet, S., Sharaiha, Y.M.: Binary digital image processing - A discrete approach. Academic Press, London (2000)

8. Ronse, C.: Minimal test patterns for connectivity preservation in parallel thinning algorithms for binary digital images. Discrete Applied Mathematics 21, 67-79 (1988)

9. Staunton, R.C.: An Analysis of Hexagonal Thinning Algorithms and Skeletal Shape Representation. Pattern Recognition 29, 1131-1146 (1996)

10. Staunton, R.C.: One-pass parallel hexagonal thinning algorithm. In: Proc. IEE 7th Int. Conf. Image Processing and Its Applications, pp. 841-845 (1999)

11. Suen, C.Y., Wang, P.S.P. (eds.): Thinning methodologies for pattern recognition. Series in Machine Perception and Artificial Intelligence, vol. 8. World Scientific, Singapore (1994)

12. Wiederhold, P., Morales, S.: Thinning on quadratic, triangular, and hexagonal cell complexes. In: Brimkov, V.E., Barneva, R.P., Hauptman, H.A. (eds.) IWCIA 2008. LNCS, vol. 4958, pp. 13-25. Springer, Heidelberg (2008) 